\title{
LIE ALGEBRAS OF VECTOR FIELDS AND GENERALIZED FOLIATIONS
}

\author{
JANUSZ GRABOWSKI
}

\begin{abstract}
The main result is a Pursell-Shanks type theorem describing isomorphism of the Lie algebras of vector fields preserving generalized foliations. The result includes as well smooth as real-analytic and holomorphic cases.
\end{abstract}

\section{Introduction.}

A whole series of papers followed the classical result of Shanks and Pursell [15] which states that the Lie algebra $\mathcal{X}(M)$ of all smooth vector fields on a smooth manifold $M$ determincs the smooth structure of $M$, i.e. the Lie algebras $\mathcal{X}\left(M_{1}\right)$ and $\mathcal{X}\left(M_{2}\right)$ are isomorphic if and only if $M_{1}$ and $M_{2}$ are diffeomorphic. Some of these papers concern special geometric situations (hamiltonian, contact, group invariant, etc. vector fields), as for example the results of Omori [14, Chapter X], Abe [1], or Atkin and Grabowski [3], and for which specific tools were developed in each case. There is however a case when the answer is more or less complete in the whole generality. These are the Lic algebras of vector fields which are modules over the corresponding rings of functions (we shall call them modular). Let us recall the work of Amemiya [2], our paper [5] where developed algebraic approach made possible to consider analytic cases as well, and finally the brilliant purely algebraic result of Skriabin [16]. This final result states that, in case when modular Lie algebras of vector fields contain finite families of vector fields with no common zeros (we shall say that they are strongly non-singulor), isomorphisms between them are generated by isomorphisms of corresponding algebras of functions, i.e. diffeomorphisms of underlying manifolds. The siandard model of a modular Lie algebra of vector fields is the Lie algebra $\mathcal{X}(\mathcal{F})$

This research was supported in 1993 by KBN, project No. 2 P301 04603. 
of all vector fields tangent to a given (generalized) foliation $\mathcal{F}$. However, if we consider not the Lie algebra of leaf preserving vector fields $\mathcal{X}(\mathcal{F})$ but the Lie algebra of foliation preserving vector fields $\mathcal{L}(\mathcal{F})$ (i.e. vector fields which generate local flows mapping leaves into leaves), it is no longer modular, since vector fields of $\mathcal{L}(\mathcal{F})$ have transversal parts "constant" along leaves (see Lemma 1 in Chapter 4), so that the general "modular" methods fail. It makes the description of isomorphisms a little bit harder and only partial solutions for smooth classical (regular) foliations were found (cf. Fukui and Tomita [4] and Rybicki [19]). In this note we present a purcly algebraic approach to this question and prove the Shanks-Pursell type result for the Lie algebras of vector fields prescrving foliations not only for classical, but also for a large class of generalized foliations. The result includes as well smooth as real-analytic and holomorphic cases.

\section{Statement of the main result.}

Since we shall be interested mainly in certain algebraic properties of the objects in question, we shall deal at the same time with finite dimensional manifolds $M$ of different classes of smoothness $\mathcal{C}: \mathcal{C}=C^{\infty}, C^{\omega}, \mathcal{H}$, where $C^{\infty}$ denotes the classical smooth case, $C^{\omega}$-analytic case, and $\mathscr{H}$ denotes holomorphic case for Stein manifolds. For details we refer to [3]. For instance, $\mathcal{C}(M)$ is the algebra of class $\mathcal{C}$ functions on the manifold $M$ of class $C$. Note that the algebras $C^{\infty}(M)$ and $C^{\omega}(M)$ are real and the algebra $\mathcal{H}(M)$ of holomorphic functions on the Stein manifold $M$ is complex. It is well-known that the corresponding Lie algebra $\mathcal{X}(M)$ of all class $C$ vector fields can be regarded as the Lie algebra of derivations of $\mathcal{C}(M)$ (in analytic cases we refer to $[6]$ ).

Definition. A generalized foliation $\mathcal{F}=\left\{\mathcal{F}^{\alpha}\right\}_{\alpha \in \Lambda}$ on a manifold $M$ of class $\mathcal{C}$ is a partition of $M$ into connected submanifolds $M=\bigcup_{a \in \Lambda} \mathcal{F}^{\alpha}$ which are cxactly the orbits of compositions of flows generated by local vector fields of class $\mathcal{C}$ tangent to the leaves of $\mathcal{F}$.

In other words, leaves of a generalized foliation consist of maximal integral manifolds of an involutive gencralized distribution $P \subset T M$ of class $\mathcal{C}$ which is invariant with respect to the flows of local vector fields with values in $P$ (cf. [17]). Note also that in analytic cases the assumption about invariance is superfluous (cf. [13]).

Generalized foliations will be called further simply foliations, while the classical foliations will be called regular foliations, since the dimension of leaves is constant. Denote by $\mathcal{X}(\mathcal{F})$ the Lie algebra of vector fields tangent to the leaves of $\mathcal{F}$ (leaf preserving vector fields). This Lie algebra is modular, i.e. it has the natural structure of an $\mathcal{C}(M)$-module. Consider 
the Lie normalizer

$$
N(\mathcal{F})=\{X \in \mathcal{X}(M):[X, \mathcal{X}(\mathcal{F})] \subset \mathcal{X}(\mathcal{F})\}
$$

of $\mathcal{X}(\mathcal{F})$ in $\mathcal{X}(M)$.

Remark 1. A "standard" normalizer consists of foliation preserving vector fields, but in the smooth case it can be larger. Consider for

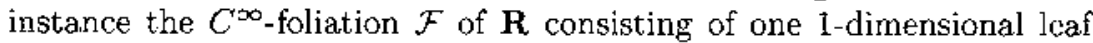
$(-\infty, 0)$ and non-negative points being 0 -dimensional leaves. It is clcar that $N(\mathcal{F})=\mathcal{X}(\mathbf{R})$, but not all smooth vector fields (e.g. generating translations) are foliation preserving.

Given $p \in M$ denote by the $\mathcal{F}_{p}$ the leaf containing $p$ and by $\mathcal{F}(p)$ the tangent space $T_{p} \mathcal{F}_{p}$. For points of $M$ the obvious equivalence relation " " means that $q \in \mathcal{F}_{p}$ ( $p$ and $q$ belong to the same leaf of $\mathcal{F}$ ).

Definition. We call a foliation $\mathcal{F}$ finitely generated if the $\mathcal{C}(M)$ module $\mathcal{X}(\mathcal{F})$ is generated by a finite family of vector fields which span $\mathcal{F}(p)$ at every $p \in M$ and non-singular if the leaves of $\mathcal{F}$ are at least one-dimensional.

It is not hard to prove the following (cf. [7] or [8]).

Theorem 1. Regular foliations of class $\mathcal{C}$ are finitely generated.

Note that all foliations generated by hamiltonian vector fields of local Lie algebras of Kirillov (cf. [11]) or, in other terminology, generated by Jacobi or Poisson structures (cf. [9]) are finitely generated.

Our main result is the following.

Theorem 2. Let $\mathcal{F}_{i}$ be a finitely generated non-singular foliation on a manifold $M_{i}$ of class $\mathcal{C}$ and let $\mathcal{L}_{i}$ be a Lie subalgebra of $N\left(\mathcal{F}_{i}\right)$ including $\mathcal{X}\left(\mathcal{F}_{i}\right)$ (e.g. $\mathcal{L}_{i}=\mathcal{L}\left(\mathcal{F}_{i}\right)$-the Lie algebra of class $\mathcal{C}$ foliation preserving vector fields), $i=1,2$. If $\Phi: \mathcal{L}_{1} \longrightarrow \mathcal{L}_{2}$ is a Lie algebra isomorphism then $\Phi=\phi_{*}$ for a foliation preserving diffeomorphism $\phi: M_{1} \longrightarrow M_{2}$ of class $\mathcal{C}$.

The above result may be reduced to the following.

Theorem 3. Under the assumptions of Theorem 2 every isomorphism $\Phi: \mathcal{L}_{1} \longrightarrow \mathcal{L}_{2}$ maps $\mathcal{X}\left(\mathcal{F}_{1}\right)$ into $\mathcal{X}\left(\mathcal{F}_{2}\right)$.

It suffices to apply Theorem 5.5 of [5] or Theorem 3.2 of [16] to see that every isomorphism $\Phi: \mathcal{X}\left(\mathcal{F}_{1}\right) \longrightarrow \mathcal{X}\left(\mathcal{F}_{2}\right)$ is implemented by a foliation preserving diffeomorphism. 
Romark that non-regular foliations have not to be finitely generated as for example the foliation from Remark 1 or the $C^{\infty}$-foliation

$$
\mathcal{F}=\left\{\mathbf{R} \backslash\left\{0,1, \frac{1}{2}, \frac{1}{3}, \ldots\right\},\{0\},\{1\},\left\{\frac{1}{2}\right\},\left\{\frac{1}{3}\right\}, \ldots\right\}
$$

of $\mathbf{R}$, but this assumption seems to be rather technical. However, in analytic cases we do not even know whether the Lie algebra $\mathcal{X}(\mathcal{F})$ is not trivial. We believe yes and due to the Theorem A of Cartan and analogous result of Tognoli [18] for real-analytic case it suffices to prove the following.

Conjecture. The analytic sheaf of germs of analytic (real or complex) vector fields tangent to a given analytic foliation is locally finitely generated.

\section{Algebraic preparation.}

Throughout this section $A$ denotes an associative commutative unital algebra over a field $k$ of characteristic $\neq 2$ and $\mathcal{X}$ denotes a subalgebra of the Lie algebra $\operatorname{Der}(A)$ of derivations of $A$ with the commutator bracket. Note that $\operatorname{Der}(A)$ is an $A$-module in the obvious way so that we have the identity

$$
[X, f Y]=X(f) Y+f[X, Y]
$$

for all $X, Y \in \operatorname{Der}(A), f \in A$.

Definition. We call $\mathcal{X} \subset \operatorname{Der}(A)$ modular if $\mathcal{X}$ is an $A$-submodule of $\operatorname{Der}(A)$ and strongly nowhere vanishing if $\mathcal{X}(A)=A$ (where clearly $\mathcal{X}(A)=\operatorname{span}\{X(f): X \in \mathcal{X}$ and $f \in A\})$.

The last property may be written in the homological way as $H_{0}(\mathcal{X}, A)=0$. Our standard model is of course $A=\mathcal{C}(M)$-the algebra of class $\mathcal{C}$ functions on the manifold $M$ and $\mathcal{X}=\mathcal{X}(\mathcal{F})$-the Lie algebra of class $\mathcal{C}$ vector fields on $M$ preserving leaves of the foliation $\mathcal{F}$. The Lic algcbra $\mathcal{X}(\mathcal{F})$ is clearly modular and it is strongly non-singular if and only if it contains a finite number of vector fields with no common zeros (cf. [5]). This is the case of $\mathcal{F}$ being finitely generated and non-singular.

Remark that a modular Lie algebra of vector fields is (in a little bit more general setting) called sometimes also a differential Lie algebra (cf. [14]).

By $\mathcal{M}(A)$ denote the set of all finite codimensional maximal ideals of $A$ and by $\mathcal{M}(\mathcal{X})$ the set of all finite codimensional maximal Lie subalgebras of $\mathcal{X}$.

It is well-known that in casc of $A=\mathcal{C}(M)$ we have $\mathcal{M}(A) \simeq M$, where the correspondence is given by

$$
M \ni p \longmapsto J(p)=\{f \in \mathcal{C}(M): f(p)=0\} \in \mathcal{M}(A)
$$


(cf. [5, Proposition 3.5]).

For $I \subset A$ put $\mathcal{X}_{I}:=\{X \in \mathcal{X}: X(A) \subset I\}, V(I):=\{J \in \mathcal{M}(A)$ : $I \subset J\}, \hat{I}:=\bigcap_{J \in V(I)} J$, and $I^{n}:=\operatorname{span}\left\{f_{1} \cdot \ldots \cdot f_{n}: f_{\perp}, \ldots, f_{n} \in I\right\}$. For $L \subset \mathcal{X}$ put $V(L):=\{K \in \mathcal{M}(\mathcal{X}): L \subset K\}$.

Due to [5, Theorem 5.1] elements of $\mathcal{M}(\mathcal{X}(\mathcal{F}))(\mathcal{F}$ finitely generated and non-singular) are of the form

$$
\mathcal{X}(\mathcal{F})_{p}:=\{X \in \mathcal{X}(\mathcal{F}): X(p)=0\}
$$

for certain $p \in M$. However, for our purposes we shall need a little bit stronger result. Its algebraic version is the following.

Theorem 4. Let $\mathcal{X}$ be a modular strongly non-singular Lie subalgebra of $\operatorname{Der}(A)$. Then for any $k=1,2,3, \ldots$ there exists $n(k)$ such that for any Lie subalgebra $L$ of $\mathcal{X}$ of codimension $\leq k$ we have $I \mathcal{X} \subset L \subset \mathcal{X}_{\tilde{I}}$ for an ideal $I$ of $A$ of codimension $\leq n(k)$.

Proof: Since $\mathcal{X}(A)=A$, therc are $X_{1}, \ldots, X_{m} \in \mathcal{X}$ and $f_{1}, \ldots, f_{m} \in A$ such that $\sum_{i=1}^{m} X_{i}\left(f_{i}\right)=1$. Put $n(k)=2 m\left(k+k^{2}\right)$. If $L$ is a Lie subalgebra of codimension $\leq k$, then $U:=\{X \in L:[X, \mathcal{X}] \subset L\}$ is a Lie subalgebra of $\mathcal{X}$ of codimension $\leq\left(k+k^{2}\right)$ as the kernel of the adjoint representation of $L$ in $\mathcal{X} / L$.

Put

$$
W:=\left\{f \in A: f X_{i} \in U \text { ond } f f_{i} X_{i} \in U, i=1, \ldots, m\right\} .
$$

Since $\operatorname{dim}(A / W) \leq 2 m\left(k+k^{2}\right)=n(k), J:=A W=\operatorname{span}\{g f: g \in$ $A, f \in W\}$ is an ideal of $A$ of codimension $\leq n(k)$. For $X \in \mathcal{X}$ and $f \in W$ the brackets $\left[f X_{i}, f_{i} X\right]$ and $\left[X, f f_{i} X_{i}\right]$ belong to $L$, so calculating their sum with the help of (3.1) we get $f X_{i}\left(f_{i}\right) X+f X\left(f_{i}\right) X_{i} \in L$.

Since $\mathcal{X}=A \mathcal{X}$, we conclude that

$$
J\left(X_{i}\left(f_{i}\right) X+X\left(f_{i}\right) X_{i}\right) \subset L \text { for all } X \in \mathcal{X}, i=1, \ldots, m .
$$

In particular,

$$
J X_{i}\left(f_{i}\right) X_{i} \subset L \text { for } i=1, \ldots, m \text {. }
$$

On the other hand, putting $X:=X_{i}\left(f_{i}\right) X$ in (3.2), we get

$$
J\left(\left(X_{i}\left(f_{i}\right)\right)^{2} X+X_{i}\left(f_{i}\right) X\left(f_{i}\right) X_{i}\right) \subset L
$$

and due to (3.3)

$$
J\left(X_{i}\left(f_{i}\right)\right)^{2} X \subset L \text { for all } X \in \mathcal{X}, i=1, \ldots, m .
$$


Since $\sum_{i=1}^{m} X_{i}\left(f_{i}\right)=\mathbf{1}$, the ideal generated by $\left\{\left(X_{i}\left(f_{i}\right)\right)^{2}: i=1, \ldots, m\right\}$ equals $A$ and (3.4) implies finally $J \mathcal{X} \subset L$. The ideal $I:=\{f \in A$ : $f \mathcal{X} \subset L\}$ includes $J$ and is therefore of codimension $\leq n(k)$. Clearly $I \mathcal{X} \subset L$ and $L$ is a Lie algebra, so in view of (3.1)

$$
L(I) \mathcal{X} \subset[L, I \mathcal{X}]+I[L, \mathcal{X}] \subset L
$$

and hence $L(I) \subset I$. This in turn implies $L(A) \subset \hat{I}$ as shows Lemma 4.2 in [5].

Coroliary 1. Every $L \in \mathcal{M}(\mathcal{X})$ is of the form $\mathcal{X}_{J}$ for a unique $J \in$ $\mathcal{M}(A)$.

The proof is straightforward.

Corollary 2. Given $B \subset \mathcal{M}(A)$ and $k=1,2 \ldots$ there is $n(k)$ such that $(\cap B)^{n(k)} \mathcal{X} \subset L$ for every at most $k$ codimensional Lie subalgebra $L$ of $\mathcal{X}$ salisfying $V(L) \subset\left\{\mathcal{X}_{J}: J \in B\right\}$.

Proof: Take $\mathrm{L}$ as above. According to Theorem 4 there is $n(k)$ and an ideal $I$ of $A$ of codimension $\leq n(k)$ such that $I \mathcal{X} \subset L$ and $L(A) \subset \bar{I}$. Since $V(L) \subset\left\{\mathcal{X}_{J}: J \in B\right\}$ implies $V(L(A)) \subset B$, we have $\bigcap B \subset \hat{I}$. Because of the codimension of $I$, the descending series $A \supset(I+\hat{I}) \supset(I+$ $\left.(\hat{I})^{2}\right) \supset \ldots$ stabilizes at at most $n(k)$-th step, so $I+(\hat{I})^{n(k)}=I+(\hat{I})^{n(k)+1}$ and by the Nakayama's Lemma $(\hat{I})^{n(k)} \subset I$, i.e. $(\bigcap B)^{n(k)} \mathcal{X} \subset I \mathcal{X} \subset$ $L$.

Theorem 5. Given finitely generated non-singular foliation $\mathcal{F}$ on a manifold $M$ of class $\mathcal{C}$ and a positive integer $k$ the intersection $\bigcap_{\alpha \in \Lambda} L_{\alpha}$ of a family of at most $k$ codimensional Lie subalgebras of $\mathcal{X}(\mathcal{F})$ is finite codimensional if $V\left(L_{\alpha}\right)=V\left(L_{\beta}\right)$ for all $\alpha, \beta \in \Lambda$.

Proof: According to Corollary 1 and our remarks at the beginning of this section, $V\left(L_{\alpha}\right)=\left\{\mathcal{X}(\mathcal{F})_{p_{i}}: i=1, \ldots, r\right\}$ for some $p_{1}, \ldots, p_{r} \in M$ and all $\alpha \in \Lambda$. Due to Corollary 2, we have the inclusion $J^{n(k)} \mathcal{X}(\mathcal{F}) \subset$ $\bigcap_{\alpha \in \Lambda} L_{\alpha}$ for $J=\bigcap_{i=1}^{T} J\left(p_{i}\right)$ being the ideal of functions vanishing at $p_{1}, \ldots, p_{r}$. This ideal is clearly finite codimensional and one can see that $J^{n(k)}$ is finite codimensional as well (cf. Note 1.4 in [6] or [10] for $C^{\infty}$ case). The $\mathcal{C}(M)$-module $\mathcal{X}(\mathcal{F})$ is finitely generated, so $J^{n(k)} \mathcal{X}(\mathcal{F})$ and hence $\bigcap_{\alpha \in \Lambda} L_{\alpha}$ is finite codimensional. 


\section{Proof of Theorem 3.}

Suppose now that $\mathcal{F}$ is a finitely generated non-singular foliation on a manifold $M$ of class $\mathcal{C}$. For any Lie algebra $L$ of vector fields on $M$ and any $p \in M$ denote $L_{p}:=\{X \in L: X(p)=0\}, L_{p}^{\perp}:=\{X \in L: X(p) \in$ $\mathcal{F}(p)\}, L(p):=\{X(p): X \in L\}$.

Recall that $N(\mathcal{F})$ is the Lie normalizer of $\mathcal{X}(\mathcal{F})$ in $\mathcal{X}(M)$ and that " $\sim$ " is the equivalence relation given by $\mathcal{F}$.

Lemma 1. If $p \sim q$, then $N(\mathcal{F})_{p}^{\perp}=N(\mathcal{F})_{q}^{\perp}$.

In other words, a vector field of $L$ is tangent to the whole leaf if at one point.

Proof: Take $Y \in N(\mathcal{F})_{p}^{\perp}$ and $X \in \mathcal{X}(\mathcal{F}), X(p) \neq 0$. Choosing local coordinates $\left(x_{1}, \ldots x_{n}\right)$ at $p \in M$ such that $X=\frac{\partial}{\partial x_{1}}$ and $x_{r+1}=\ldots=x_{n}=0$ describes locally a component of $\mathcal{F}_{p}$, we can write $Y=\sum_{i=1}^{t i} f_{i}(x) \frac{\partial}{\partial x_{i}}$, where $f_{i}(p)=f_{i}(0)=0$ for $i=r+1, \ldots, n$. Since the vector field $[X, Y]=$ $\sum_{i=1}^{n} \frac{\partial f_{i}}{\partial x_{1}} \frac{\partial}{\partial x_{i}}$ belongs to $\mathcal{X}(\mathcal{F})$, we have $\frac{\partial f_{2}}{\partial x_{1}}\left(x_{1}, \ldots, x_{r}, 0, \ldots, 0\right)=0$ for $i=r+1, \ldots, n$. It shows that $f_{i}, i=r+1, \ldots, n$, are constant (and hence $=0$ ) along trajectories of $\mathcal{X}(\mathcal{F})$ through $p$, so $Y$ is tangent to the leaf $\mathcal{F}_{p}$ at any its point.

Lemma 2. If $L$ is a Lie ideal of $N(\mathcal{F})$ with $L(p) \not \subset \mathcal{F}(p)$ for certain $p \in M$, then for any $q \sim p$ we have $\mathcal{F}(q) \subset L(q)$ and $V\left(L_{q}\right)=V\left(L_{p}\right)$, where $V(L, q)=\left\{K \in \mathcal{M}(L): L_{q} \subset K\right\}$. Moreover $\bigcap_{q \in \mathcal{F}_{p}} L_{q}$ is infinite codimensional in $L$.

Proof: Take $Y \in L$ with $Y(p) \notin \mathcal{F}(p)$. According to Lemma 1 we have $Y(q) \notin \mathcal{F}(q)$ for any $q \in \mathcal{F}_{p}$. Given a finite set $\left\{q_{1}, \ldots, q_{s}\right\}$ of points of $\mathcal{F}_{p}$ we can find $f \in \mathcal{C}(M)$ vanishing at $q_{1}, \ldots, q_{s}$ and such that. $Y(f)\left(q_{i}\right)=a_{i}, i=1, \ldots, s$, are arbitrarily chosen. For any $X \in \mathcal{X}(\mathcal{F})$ the vector field $[Y, f X]$ belongs to $L$. Since $[Y, f X]\left(q_{i}\right)=a_{i} X\left(q_{i}\right)$, the intersection $\bigcap_{q \in \mathcal{F}_{p}} L_{q}$ is infinite codimensional and $\mathcal{F}(q) \subset L(q)$ for any $q \in \mathcal{F}_{p}$. Take now $K \in V\left(L_{q}\right)$. We claim that $L_{q}^{\perp} \subset K$. Indeed, $L_{q}^{\perp}$ is a. Lie subalgebra including $L_{q}$ and $L_{q}^{\perp} \not \subset K$ means that $\mathcal{F}(q) \not \subset K(q)$. $K(q) \subset \mathcal{F}(q)$ would imply that $K \subset L_{q}^{\perp}$, but by the assumption $L_{q}^{\perp} \neq L$, so by the maximality of $K$ we would have $K=L_{q}^{+}$and $\mathcal{F}(q) \subset K(q)$. Assume therefore that there is $Z \in K, Z(q) \notin \mathcal{F}(q)$. Since $L$ is a Lie idcal of $N(\mathcal{F}),[Y, f X] \in L_{q}$ and hence $[Z,[Y, f X]] \in K$ for any $X \in \mathcal{X}(\mathcal{F})$ and any $f \in \mathcal{C}(M)$ vanishing at $q$ and such that $Y(f)(q)=0$. Chosing such an $f$ satisfying additionally $Z(f)(q)=0$ and $Z(Y(f))(q)=1$ we have $[Z,[Y, f X]](q)=X(q)$ and hence $\mathcal{F}(q) \subset K(q)$. Therefore $V\left(L_{q}\right)=$ 
$\left\{K \in \mathcal{M}(L): L_{q}^{\perp} \subset K\right\}$ and since $L_{q}^{\perp}=L_{p}^{\perp}$, we get $V\left(L_{q}\right)=V\left(I_{p}\right)$ for $q \sim p$.

Proof of Theorem 3: Suppose $L=\Phi\left(\mathcal{X}\left(\mathcal{F}_{1}\right)\right)$ is not included in $\mathcal{X}\left(\mathcal{F}_{2}\right)$. Then $\mathrm{L}$ is a Lic ideal of $N\left(\mathcal{F}_{2}\right)$ with $L(p) \not \subset \mathcal{F}_{2}(p)$ for certain $p \in M_{2}$. According to Lemma $2, V\left(L_{q}\right)=V\left(L_{p}\right)$ for all $q \in\left(\mathcal{F}_{2}\right)_{p}$ and $\bigcap_{q \in\left(\mathcal{F}_{2}\right)_{p}} L_{q}$ is infinite codimensional. On the other hand, $\mathrm{L}$ is isomorphic to $\mathcal{X}\left(\mathcal{F}_{1}\right)$ and due to Theorem 5 this intersection should be of finite codimension, since $\operatorname{dim}\left(L / L_{q}\right) \leq \operatorname{dim}\left(M_{2}\right)$.

\section{References}

1. K. ABE, Pursell-Shanks type theorem for orbit spaces of $G$-manifolds, Publ. Res. Inst. Moth. Sci. 18 (1982), 265-282.

2. I. AмемIYA, Lie algebra of vector fields and complex structure, $J$. Math. Soc. Japan 27 (1975), 545-549.

3. C. J. ATKIN AND J. Grabowski, Homomorphisms of the Lie algebras associated with a symplectic manifold, Compos. Math. 76 (1990), 315-349.

4. K. FuKui ANd N. TOMiTA, Lie algebra of foliation preserving vector fields, J. Math. Kyoto Univ. 22 (1982), 685-699.

5. J. GRABOWSKI, Isomorphisms and ideals of the Lie algebras of vector fields, Invent. Math. 50 (1978), 13-33.

6. J. GraBowsKI, Derivations of the Lie algebras of analytic vector ficlds, Compos. Math. 43 (1981), 239-252.

7. I. GRABOWSKI, The Lie algebras of vector fields on manifolds, thesis, Warsaw University, 1981, (Polish).

8. J. GRABOWSKI, Ideals of the Lie algebras of vector fields revisited, to appear in Geom. and Phys., Proc. 12th Winter School, Srni 1992.

9. F. Guedira AND A. Lichnerowicz, Géométrie des algèbres de Lie locales de Kirillov, J. Math. Pures Appl. 63 (1984), 407-484.

10. G. KAINZ AND P. MiCHOR, Natural transformations in differential geometry, Czech. Math. J. 37 (1987), 584-607.

11. A. A. Kirillov, Local Lie algebras, Russ. Math. Surv. 31, No 4 (1976), 5575.

12. Y. Kosmann-Schwarzbach and F. Magri, Poisson-Nijenhuis structures, Ann. Inst. H. Poincaré Phys. Theor. 53 (1990), 35-81.

13. T. NAGANO, Linear differential systems with singularities and an application to transitive algebras, J. Math. Soc. Jopan 18 (1966), $398 \cdots 404$. 
14. H. OMORI, "Infinite Dimensional Lie Transformation Groups," Lect. Notes in Math. 427, Springer Verlag, 1976.

15. M. E. Shanks Aind L. E. Pursell, The Lie algebra of a smooth manifold, Proc Amer. Math. Soc. 5 (1954), 468.472.

16. S. M. SkRIABIN, The regular Lie rings of derivations of commutative rings, preprint WINITI 4403-W87 (1987), (Russian).

17. H. J. Sussian, Orbits of families of vector ficlds and integrability of distributions, Trans. Amer. Math. Soc. 180 (1973), 151-158.

18. A. Tognoli, Some results in the theory of real analytic spaces, in "Espaces Analytiques," (Séminaire Bucharest 1969), Ed. Acad. R. S. R., Bucharest, 1971, pp. 149-157.

19. T. RYBICKI, Lie algebras of vector fields and codimension one foliation, Publ. Mat. (UAB) 34 (1990), 311-321.

Institute of Mathematics

University of Warsaw

ul. Banacha 2

PL 02-097 Warsaw

POLAND

Primera versió rebuda el 19 de Febrer de 1993, darrera versió rebuda el 30 de Setembre de 1993 